Bull. Korean Math. Soc. 51 (2014), No. 5, pp. 1539-1549

http://dx.doi.org/10.4134/BKMS.2014.51.5.1539

\title{
ON THE STABILITY OF SPACELIKE HYPERSURFACES WITH HIGHER ORDER MEAN CURVATURE IN A DE SITTER SPACE
}

\author{
Shicheng Zhang
}

\begin{abstract}
The closed spacelike hypersurfaces with higher order mean curvature is discussed in a de Sitter space. The hypersurface is proved stable if and only if it is totally umbilical.
\end{abstract}

\section{Introduction}

As we all know, the hypersurfaces with constant mean curvature or constant scalar curvature in real space forms are characterized as critical points of the area functional for volume-preserving variations. Many results have been achieved about hypersurfaces with constant mean curvature or constant scalar curvature in a unit sphere $S^{n+1}(1)[1,2,3]$. Among these results, the geodesic sphere is the only stable compact hypersurface with constant mean curvature in a sphere as in [3]. After that, the closed hypersurfaces with higher order mean curvature immersed in a Riemannian space form are studied and similar results are obtained by other researches $[4,8,12]$.

Achievements are not only obtained in Riemannian space, in fact, many researches are also conducted in Lorentzian spaces. Constant mean curvature spacelike hypersurfaces are solutions to a variational problems. Actually, they are the critical points of the area functional for variations that leave constant a certain volume function. In this sense, Barbosa and Oliker [5] computed the second variation formula and obtained in the de Sitter space $S_{1}^{n+1}$ that spheres maximize the area functional for volume-preserving variations, which is consistent with the definition of stability. Later, researches of $[6,11]$, they obtained an extension of the result in [5] for spacelike hypersurfaces with constant scalar curvature, respectively.

Motivated by works [7, 9], The stability of closed spacelike hypersurfaces in a de Sitter space $S_{1}^{n+1}$ is considered in this paper. This concept arises from considering the variational problem of minimizing a suitable linear combination

Received August 31, 2013.

2010 Mathematics Subject Classification. 53C42, 53A10.

Key words and phrases. de Sitter space, spacelike hypersurface, stability, higher order mean curvature. 
of the 2 nd area for volume-preserving variations. Therefore, the purpose of this paper is to prove that a closed spacelike hypersurface in the de Sitter space $S_{1}^{n+1}$ is stable if and only if it is totally umbilical. Precisely, the following result is to be obtained.

Theorem 1.1. Let $M$ be a closed orientable hypersurface in de Sitter space $S_{1}^{n+1}$ satisfying $b_{r} H_{r+1}-a n H=b$ for some constants $a \leq 0$ and $b$. By choosing the suitable orientation, we assume that $H>0$. Then $M$ is stable if and only if $M$ is totally umbilical. Where $b_{r}=(n-r)\left(\begin{array}{l}n \\ r\end{array}\right)$ and $1 \leq r \leq n-1$.

When $r=1$, we have:

Corollary 1.1. Let $M$ be a closed orientable hypersurface in de Sitter space $S_{1}^{n+1}$ satisfying $(n-1) H_{2}-a H=b$ for some constants $a \leq 0$ and $b$. By choosing the suitable orientation, we assume that $H>0$. Then $M$ is stable if and only if $M$ is totally umbilical.

When $a=0$, we have the Corollary 1.2 in [7]:

Corollary 1.2. Let $M$ be a closed orientable hypersurface with constant higher order mean curvature in de Sitter space $S_{1}^{n+1}$ and $H_{r}>0$. Then $M$ is stable if and only if $M$ is totally umbilical.

When $r=1$ and $a=0$, we have the main theorem in [11]:

Corollary 1.3. Let $M$ be a closed orientable hypersurface with constant scalar curvature in de Sitter space $S_{1}^{n+1}$. Then $M$ is stable if and only if $M$ is totally umbilical.

Remark 1.1. Comparing with the main theorem in $[5,11]$, we withdraw the constant mean curvature or constant scalar curvature and obtain the same result.

\section{Preliminaries}

For what follows, we recall that the $(n+2)$-dimensional Lorentz-Minkowski space $\mathbb{R}_{1}^{n+2}$ is the real vector space $\mathbb{R}^{n+2}$ endowed with the Lorentz metric

$$
\langle\nu, \omega\rangle=-\nu_{0} \omega_{0}+\sum_{i=1}^{n+1} \nu_{i} \omega_{i}
$$

for all $\nu, \omega \in \mathbb{R}^{n+2}$. The $(n+1)$-dimensional de Sitter space $S_{1}^{n+1}$ is given by

$$
S_{1}^{n+1}=\left\{p \in R_{1}^{n+2}:\langle p, p\rangle=1\right\} .
$$

The induced metric from $\langle$,$\rangle makes S_{1}^{n+1}$ into a Lorentz manifold with constant sectional curvature one. Moreover, if $p \in S_{1}^{n+1}$, we can put

$$
T_{p}\left(S_{1}^{n+1}\right)=\left\{\nu \in R_{1}^{n+2}:\langle\nu, p\rangle=0\right\} .
$$


A smooth immersion $x: M \rightarrow S_{1}^{n+1} \hookrightarrow \mathbb{R}_{1}^{n+2}$ of an $n$-dimensional connected manifold $M$ is said to be a spacelike hypersurface if the induced metric via $x$ is a Riemannian metric on $M$, which, as usual, is also denoted by $\langle$,$\rangle .$

Observe that $e_{n+1}=\{0, \ldots, 0,1\}$ is a unit timelike vector field globally defined on $\mathbb{R}_{1}^{n+2}$, which determines a time-orientation on $\mathbb{R}_{1}^{n+2}$. Thus we can choose a unique timelike unit normal field $N$ on $M$ which is past-directed on $\mathbb{R}_{1}^{n+2}$ (i.e., $\left\langle N, e_{n+1}\right\rangle>0$ ), and hence we may assume that $M$ is oriented by $N$.

Let $x: M \rightarrow S_{1}^{n+1} \hookrightarrow \mathbb{R}_{1}^{n+2}$ be an immersed spacelike hypersurface in de Sitter $S_{1}^{n+1}$, and let $N$ be its past-directed timelike normal field. In order to set up the notation, we will denote by $\nabla^{0}, \bar{\nabla}$ and $\nabla$ the Levi-Civita connections of $\mathbb{R}_{1}^{n+2}, S_{1}^{n+1}$ and $M$, respectively. Then the Gauss and Weingarten formulae for $M$ in $S_{1}^{n+1} \hookrightarrow \mathbb{R}_{1}^{n+2}$ are given respectively by

$$
\begin{aligned}
\nabla_{V}^{0} W & =\bar{\nabla}_{V} W-\langle V, W\rangle x \\
& =\nabla_{V} W-\langle A V, W\rangle N-\langle V, W\rangle x
\end{aligned}
$$

and

$$
A(V)=-\nabla_{V}^{0} N=-\bar{\nabla}_{V} N
$$

for all tangent vector fields $V, W \in \mathcal{X}(M)$, where $A$ stands for the shape operator of $M$ in $S_{1}^{n+1}$ associated with $N$.

At each $p \in M$, A restricts to a self-adjoint linear map $A_{p}: T_{p} M \rightarrow T_{p} M$. For $1 \leq r \leq n$, let $S_{r}(p)$ denote the $r$-th elementary symmetric function on the eigenvalues of $A_{p}$, in this way one gets $n$ smooth functions $S_{r}: M \rightarrow \mathbb{R}$, such that

$$
\operatorname{det}(t I-A)=\sum_{k=0}^{n}(-1)^{k} S_{k} t^{n-k},
$$

where $S_{0}=1$ by definition. If $p \in M$ and $\left\{e_{k}\right\}$ is a basis of $T_{p} M$ formed by eigenvectors of $A_{p}$, corresponding with eigenvalues $\left\{\lambda_{k}\right\}$, one immediately sees that

$$
S_{r}=\sigma_{r}\left(\lambda_{1}, \ldots, \lambda_{n}\right),
$$

where $\sigma_{r} \in R\left[X_{1}, \ldots, X_{n}\right]$ is the $r$-th elementary symmetric polynomial on the indeterminates $X_{1}, \ldots, X_{n}$.

For $1 \leq r \leq n$, one defines the $r$-th mean curvature $H_{r}$ of $x$ by

$$
\left(\begin{array}{l}
n \\
r
\end{array}\right) H_{r}=(-1)^{r} S_{r}=\sigma_{r}\left(-\lambda_{1}, \ldots,-\lambda_{n}\right) .
$$

In particular, for $r=1$,

$$
H_{1}=\frac{1}{n} \sum_{k=1}^{n} \lambda_{k}=H
$$

is the mean curvature of $M$, which is the main extrinsic curvature of the hypersurface. When $r=2, H_{2}$ defines a geometric quantity which is related to the 
(intrinsic) normalized scalar curvature $R$ of the hypersurface. More precisely, it follows from the Gauss equation that

$$
R=1+H_{2} .
$$

On the other hand, with a straightforward computation we verify that

$$
|A|^{2}=n^{2} H^{2}-n(n-1) H_{2},
$$

where $|A|^{2}$ denotes the squared norm of the shape operator of $M$.

We also define, for $0 \leq r \leq n$, the $r$-th Newton transformation $P_{r}$ on $M$ by setting $P_{0}=I$ (the identity operator) and, for $1 \leq r \leq n$, via the recurrence relation

$$
P_{r}=(-1)^{r} S_{r} I-A P_{r-1} .
$$

A trivial induction shows that

$$
P_{r}=(-1)^{r}\left(S_{r} I-S_{r-1} A+S_{r-2} A^{2}-\cdots+(-1)^{r} A^{r}\right),
$$

so $P_{n}=0$ is obtained from the Cayley-Hamilton theorem. Moreover, since $P_{r}$ is a polynomial in $A$ for every $r$, it is also self-adjoint and commutes with $A$. Therefore, all bases of $T_{p} M$ diagonalizing $A$ at $p \in T_{p} M$ also diagonalize all of the $P_{r}$ at $p$. Let $\left\{e_{k}\right\}$ be such a basis. Denoting by $A_{i}$ the restriction of $A$ to $\left\langle e_{i}\right\rangle^{\perp} \subset T_{p} \Sigma$, it is easy to see that

$$
\operatorname{det}\left(t I-A_{i}\right)=\sum_{k=0}^{n}(-1)^{k} S_{k}\left(A_{i}\right) t^{n-k}
$$

where

$$
S_{k}\left(A_{i}\right)=\sum_{\substack{1 \leq j_{1}<\cdots<j_{k} \leq n \\ j_{1}, \ldots, j_{k} \neq i}} \lambda_{j_{1}} \cdots \lambda_{j_{k}} .
$$

With the above notation, it is also immediately checked that

$$
P_{r} e_{i}=(-1)^{r} S_{r}\left(A_{i}\right) e_{i},
$$

and hence (Lemma 2.1 of [4]):

Lemma 2.1. For each $1 \leq r \leq n-1$

(a) $S_{r}\left(A_{i}\right)=S_{r}-\lambda_{i} S_{r-1}\left(A_{i}\right)$;

(b) $\operatorname{tr}\left(P_{r}\right)=(-1)^{r} \sum_{i=1}^{n} S_{r}\left(A_{i}\right)=(-1)^{r}(n-r) S_{r}=b_{r} H_{r}$;

(c) $\operatorname{tr}\left(A P_{r}\right)=(-1)^{r} \sum_{i=1}^{n} \lambda_{i} S_{r}\left(A_{i}\right)=(-1)^{r}(r+1) S_{r+1}=-b_{r} H_{r+1}$;

(d) $\operatorname{tr}\left(A^{2} P_{r}\right)=(-1)^{r} \sum_{i=1}^{n} \lambda_{i}^{2} S_{r}\left(A_{i}\right)=(-1)^{r}\left(S_{1} S_{r+1}-(r+2) S_{r+2}\right)$, where $b_{r}=(n-r)\left(\begin{array}{l}n \\ r\end{array}\right)$.

Associated with each Newton transformation $P_{r}$, one has the second-order linear differential operator $L_{r}: \mathcal{C}^{\infty}(M) \rightarrow \mathcal{C}^{\infty}(M)$, given by

$$
L_{r}(f)=\operatorname{tr}\left(P_{r} \operatorname{Hess} f\right) \text {. }
$$


We remark that $L_{0}$ is the Laplacian operator $\Delta$ and $L_{1}$ is the Cheng-Yau's square operator $\square$ defined in [10]. According to [13], $P_{r}$ is a divergence-free whenever $S_{1}^{n+1}$ is of constant sectional curvature; consequently,

$$
L_{r}(f)=\operatorname{div}\left(P_{r} \nabla f\right) .
$$

Useful consequences of (2.1) are given in the following

Proposition 2.1. If $M$ is a closed Riemannian manifold or if $M$ is a noncompact Riemannian manifold and $f$ has compact support, then

$$
\int_{M} L_{r}(f) d M=0, \int_{M} f L_{r}(f) d M=-\int_{M}\left\langle P_{r} \nabla f, \nabla f\right\rangle d M .
$$

\section{The variation problem}

Let $X$ be a variation of $x: M \rightarrow S_{1}^{n+1}$, which is a differentiable map $X:(-\varepsilon, \varepsilon) \times M \rightarrow S_{1}^{n+1}, \varepsilon>0$, such that $X_{0}=x$ and for each $t \in(-\varepsilon, \varepsilon)$, $X_{t}(\cdot)=X(t, \cdot)$ is an immersion from $M$ to $S_{1}^{n+1}$, and $\left.X_{t}\right|_{\partial M}=\left.x\right|_{\partial M}$.

Next, we let $d M_{t}$ denote the volume element of the metric induced on $M$ by $X_{t}$ and $N_{t}$ the unit normal vector field along $X_{t}$.

The variational field associated with the variation $X$ is the vector field $\left.\frac{\partial X}{\partial t}\right|_{t=0}$. Let $f=-\left\langle\frac{\partial X}{\partial t}, N_{t}\right\rangle$, we get

$$
\frac{\partial X}{\partial t}=f N_{t}+\left(\frac{\partial X}{\partial t}\right)^{\top}
$$

where $\top$ stands for tangential components.

The balance of volume of the variation $X$ is the function $V:(-\varepsilon, \varepsilon) \rightarrow \mathbb{R}$ given by

$$
V(t)=\int_{[0, t] \times M} X^{*}\left(d S_{1}^{n+1}\right) .
$$

and we say $X$ is volume-preserving if $V(t)=V(0)$ for all $t \in(-\varepsilon, \varepsilon)$. The following lemma is classical (cf. [11]).

Lemma 3.1. Let $\bar{M}^{n+1}$ be a time-oriented Lorentz manifold and $x: M \rightarrow$ $\bar{M}^{n+1}$ a closed spacelike hypersurface. If $X: M \times(-\varepsilon, \varepsilon) \rightarrow \bar{M}^{n+1}$ is a variation of $x$, then

$$
\left.\frac{d V}{d t}\right|_{t=0}=\int_{M} f d M .
$$

In particular, $X$ is volume-preserving if and only if $\int_{M} f d M_{t}=0$ for all $t$.

In order to extend [4] to the Lorentz setting, we define the $r$-area functional $\mathcal{A}_{r}(t):(-\varepsilon, \varepsilon) \rightarrow \mathbb{R}$ associated with the variation $X$ by

$$
\mathcal{A}_{r}(t)=\int_{M} F_{r}\left(S_{1}, S_{2}, \ldots, S_{r}\right) d M_{t},
$$


where $S_{r}=S_{r}(t)$ and $F_{r}$ is recursively defined by setting $F_{0}=1, F_{1}=-S_{1}$ and, for $2 \leq r \leq n-1$,

$$
F_{r}=(-1)^{r} S_{r}-\frac{c(n-r+1)}{r-1} F_{r-2} .
$$

The next step is the Lorentz analogue of Proposition 4.1 of [3]. Since it seems to us that their proof only works on a neighborhood free of umbilics, and in order to keep this work self-contained, an alternative one is presented here.

Lemma 3.2. Let $x: M \rightarrow S_{1}^{n+1}$ be a closed spacelike hypersurface of the de Sitter space $S_{1}^{n+1}$, and let $X: M \times(-\varepsilon, \varepsilon) \rightarrow S_{1}^{n+1}$ be a variation of $x$. Then,

$$
\frac{\partial S_{r+1}}{\partial t}=(-1)^{r+1}\left[L_{r} f+\operatorname{tr}\left(P_{r}\right) f-\operatorname{tr}\left(A^{2} P_{r}\right) f\right]+\left\langle\left(\frac{\partial X}{\partial t}\right)^{\top}, \nabla S_{r+1}\right\rangle .
$$

For the constant $a$, the Jacobi functional associated to the variation $X$ is given by $J:(-\varepsilon, \varepsilon) \rightarrow \mathbb{R}$

$$
J(t)=\mathcal{A}_{r}(t)-a .
$$

The following proposition is reached,

Proposition 3.1 (First Variation Formula). Let $M$ be an $n$-dimensional closed spacelike hypersurface in the de Sitter space $S_{1}^{n+1}$. For any variation of $x$ : $M \rightarrow S_{1}^{n+1}$, we have

$$
\frac{d J(t)}{d t}=\int_{M}\left[b_{r} H_{r+1}+c_{r}-a n H\right],
$$

where $c_{r}=0$ if $r$ is even and $c_{r}=-\frac{n(n-2) \cdots(n-r+1)}{(r-1)(r-3) \cdots 2}(-1)^{(r+1) / 2}$ if $r$ is odd.

Proof. From Lemma 3.2, we have

$$
\begin{aligned}
& \frac{d J(t)}{d t} \\
= & \int_{M} F_{r}^{\prime} d M_{t}+\int_{M}\left(F_{r}-a\right) \frac{\partial}{\partial t} d M_{t} \\
= & \int_{M}\left[(-1)^{r} S_{r}^{\prime}-\frac{n-r+1}{r-1} F_{r-2}^{\prime}\right] d M_{t} \\
& +\int_{M}\left[(-1)^{r} S_{r}-\frac{n-r+1}{r-1} F_{r-2}-a\right] \frac{\partial}{\partial t} d M_{t} \\
= & \int_{M}(-1)^{r}\left[S_{r}^{\prime}-S_{1} S_{r} f+S_{r} \operatorname{div}(\partial X / \partial t)^{\top}\right]+a\left[S_{1} f-\operatorname{div}(\partial X / \partial t)^{\top}\right] d M_{t} \\
& -\frac{n-r+1}{r-1} \mathcal{A}_{r-2}^{\prime} \\
= & \int_{M}\left[\operatorname{tr}\left(P_{r-1}\right) f+L_{r-1} f-\operatorname{tr}\left(A^{2} P_{r-1} f\right)+(-1)^{r}\left\langle\nabla S_{r},(\partial X / \partial t)^{\top}\right\rangle\right] d M_{t} \\
& +(-1)^{r} \int_{M}\left(-S_{1} S_{r} f+S_{r} \operatorname{div}(\partial X / \partial t)^{\top}\right) d M_{t}-\frac{n-r+1}{r-1} \mathcal{A}_{r-2}^{\prime}
\end{aligned}
$$




$$
\begin{aligned}
& +a \int_{M}\left[S_{1} f-\operatorname{div}(\partial X / \partial t)^{\top}\right] d M_{t} \\
= & \int_{M}\left[(-1)^{r-1}(n-r+1) S_{r-1} f-(-1)^{r-1}\left(S_{1} S_{r}-(r+1) S_{r+1}\right) f+a S_{1} f\right] d M_{t} \\
& \left.+(-1)^{r} \int_{M}\left\langle\nabla S_{r},(\partial X / \partial t)^{\top}\right\rangle\right] d M_{t} \\
& +\int_{M}\left[(-1)^{r+1} S_{1} S_{r} f+(-1)^{r} S_{r} \operatorname{div}(\partial X / \partial t)^{\top}\right] d M_{t} \\
& -\frac{n-r+1}{r-1} \int_{M}\left[(-1)^{r-1}(r-1) S_{r-1}+c_{r-2}\right] f d M_{t}-a \int_{M} \operatorname{div}(\partial X / \partial t)^{\top} d M_{t} .
\end{aligned}
$$

It now suffices to apply the divergence theorem. Note that $c_{r}=-\frac{n-r+1}{r-1} c_{r-2}$, then

$$
\begin{aligned}
\frac{d J(t)}{d t} & =\int_{M}\left[(-1)^{r+1}(r+1) S_{r+1}+c_{r}+a S_{1}\right] f d M_{t} \\
& =\int_{M}\left[b_{r} H_{r+1}+c_{r}-a n H\right] .
\end{aligned}
$$

By a direct application of Lemma 3.2 and Proposition 3.1, after a long but direct computation, we obtain

Proposition 3.2 (Second Variation Formula). Let $x: M \rightarrow S_{1}^{n+1}$ be a closed spacelike hypersurface satisfying $b_{r} H_{r+1}-a n H=b$, where $2 \leq r \leq n-1$, a and $b$ are constants. $X$ is a variation of $x$, then the second derivative of $J$ at $t=0$ is given by

$$
\begin{aligned}
J^{\prime \prime}(0)(f)= & \int_{M}\left\{(r+1) L_{r}-a \Delta\right) f+(r+1)\left[(-1)^{r}(n-r) S_{r}\right. \\
(3.1) \quad & \left.\left.+(-1)^{r+1}\left(S_{1} S_{r+1}-(r+2) S_{r+2}\right)\right] f+a\left(n-S_{1}^{2}+2 S_{2}\right) f\right\} f d M .
\end{aligned}
$$

Definition 3.1. Let $x: M \rightarrow S_{1}^{n+1}$ be a closed spacelike hypersurface satisfying $b_{r} H_{r+1}-a n H=b$, where $2 \leq r \leq n-1, a$ and $b$ are constants. The immersion $x$ is said to be stable if $J^{\prime \prime}(0)(f) \leq 0$ for all volume-preserving variations of $x$.

Then from the above definition, a closed spacelike hypersurface satisfying $b_{r} H_{r+1}-a n H=b$ is stable if and only if $J^{\prime \prime}(0)(f) \leq 0$ for all differentiable function $f$ which satisfies $\int_{M} f d M=0$. This can be proved following a similar argument as in $[3,11]$, the details are omitted here.

\section{Proof of theorem}

In this section, we will prove our main theorem. 
Proof of Theorem 1.1. Firstly, suppose that $M$ is totally umbilical hypersurface in $S_{1}^{n+1}$. Then the principal curvatures and $H$ are constants, we may assume $H>0$. Thus we have

$$
S_{r}=(-1)^{r}\left(\begin{array}{l}
n \\
r
\end{array}\right) H^{r}
$$

and

$$
L_{r} f=\left(\begin{array}{c}
n-1 \\
r
\end{array}\right) H^{r} \Delta f .
$$

Choose $f: M \rightarrow R$ such that $\int_{M} f d M=0$. From the second variation formula (3.1) of $J$, we have

$$
\begin{aligned}
J^{\prime \prime}(0)(f)= & \int_{M}\left\{(r+1) L_{r}-a \Delta\right) f+(r+1)\left[(-1)^{r}(n-r) S_{r}\right. \\
& \left.\left.+(-1)^{r+1}\left(S_{1} S_{r+1}-(r+2) S_{r+2}\right)\right] f+a\left(n-S_{1}^{2}+2 S_{2}\right) f\right\} f d M \\
= & \left((r+1)\left(\begin{array}{c}
n-1 \\
r
\end{array}\right) H^{r}-a\right) \int_{M}\left(n\left(H^{2}+1\right) f^{2}+f \Delta f\right) d M \\
\leq & \left((r+1)\left(\begin{array}{c}
n-1 \\
r
\end{array}\right) H^{r}-a\right) \int_{M}\left(n\left(H^{2}+1\right)-\lambda(M)\right) f^{2} d M
\end{aligned}
$$

where $\lambda(M)$ is the first eigenvalue of the Laplacian $\Delta$ in $M$. Since $M$ is totally umbilical, $M$ is sphere. Then we have $\lambda(M)=n\left(H^{2}+1\right)$. By the assumption that $H>0$ and $a \leq 0$, we obtain $J^{\prime \prime}(0)(f) \leq 0$ for all $f$ with $\int_{M} f d M=0$. Therefore it is concluded that $M$ is stable.

Now consider the reversed part. Let $M \subset S_{1}^{n+1}$ be a stable spacelike hypersurface satisfying $b_{r} H_{r+1}-a n H=b$ for some constants $a \leq 0$ and $b$. We will show that $M$ is totally umbilical.

Let $x: M \rightarrow S_{1}^{n+1} \subset \mathbb{R}_{1}^{n+2}$. Fix a unit vector $\nu \in \mathbb{R}_{1}^{n+2}$ and define functions $f$ and $g$ on $M$ by

$$
f=\langle N, \nu\rangle, g=\langle x, \nu\rangle .
$$

These are called height functions in the direction $\nu$ associated to the hypersurface. We need the following result.

Lemma 4.1. If $f$ and $g$ are the height functions of a hypersurface $x: M \rightarrow$ $S_{1}^{n+1}$ defined as above, then

$$
\begin{aligned}
L_{r}(g)= & (-1)^{r}(r+1) S_{r+1} f-(-1)^{r}(n-r) S_{r} g, \\
L_{r}(f)= & (-1)^{r+1}\left(S_{1} S_{r+1}-(r+2) S_{r+2}\right) f+(-1)^{r}(r+1) S_{r+1} g \\
& +(-1)^{r+1}\left\langle\nu, \nabla S_{r+1}\right\rangle .
\end{aligned}
$$

Proof. For a fixed arbitrary vector $\nu \in \mathbb{R}_{1}^{n+2}$, let us consider the functions $f=\langle N, \nu\rangle$ and $g=\langle x, \nu\rangle$ on $M$, we have

$$
X(\langle x, \nu\rangle)=\langle X, \nu\rangle=\left\langle X, \nu^{\top}\right\rangle,
$$




$$
X(\langle N, \nu\rangle)=-\langle A X, \nu\rangle=-\left\langle X, A\left(\nu^{\top}\right)\right\rangle
$$

for every vector field $X \in \mathcal{X}(M)$, where $\nu^{\top} \in \mathcal{X}(M)$ denotes the tangential component of $\nu$,

$$
\nu=\nu^{\top}+\langle N, \nu\rangle N+\langle x, \nu\rangle x
$$

Then the gradients of $\langle x, \nu\rangle$ and $\langle N, \nu\rangle$ on $M$ are given by $\nabla\langle x, \nu\rangle=\nu^{\top}$ and $\nabla\langle N, \nu\rangle=-A\left(\nu^{\top}\right)$, respectively. By taking covariant derivative in (4.1) and using the Gauss and Weingarten formulae, we get

$$
\nabla_{X} \nabla\langle x, \nu\rangle=\nabla_{X} \nu^{\top}=\langle N, \nu\rangle A X-\langle x, \nu\rangle X
$$

for every tangent vector field $X \in \mathcal{X}(M)$. Therefore, by Lemma 2.1, we obtain

$$
\begin{aligned}
L_{r} g & =\operatorname{tr}\left(A P_{r}\right) f-\operatorname{tr}\left(P_{r}\right) g \\
& =(-1)^{r}(r+1) S_{r+1} f-(-1)^{r}(n-r) S_{r} g \\
& =-b_{r} H_{r+1} f-b_{r} H_{r} g .
\end{aligned}
$$

On the other hand, from (4.2), we get

$$
\begin{aligned}
\nabla_{X} \nabla\langle N, \nu\rangle & =-\nabla_{X}\left(A \nu^{\top}\right) \\
& =-\nabla_{X}(A) \nu^{\top}-\langle N, \nu\rangle A^{2} X+\langle x, \nu\rangle A X .
\end{aligned}
$$

By Codazzi equation, we know that $\nabla A$ is symmetric and then

$$
\nabla A\left(\nu^{\top}, X\right)=\nabla A\left(X, \nu^{\top}\right)=\left(\nabla_{\nu^{\top}} A\right) X
$$

By (4.3) and Lemma 2.1, we obtain

$$
\begin{aligned}
L_{r} f= & -\operatorname{tr}\left(P_{r} \nabla_{\nu^{\top}} A\right)-f \operatorname{tr}\left(A^{2} P_{r}\right)+g \operatorname{tr}\left(A P_{r}\right) \\
= & (-1)^{r+1}\left\langle\nu, \nabla S_{r+1}\right\rangle+(-1)^{r+1}\left(S_{1} S_{r+1}-(r+2) S_{r+2}\right) f \\
& +(-1)^{r}(r+1) S_{r+1} g .
\end{aligned}
$$

As follows, we recall that the $(n+2)$-dimensional Lorentz-Minkowski space $\mathbb{R}_{1}^{n+2}$ is the real vector space $\mathbb{R}^{n+2}$ endowed with the Lorentz metric

$$
\langle\nu, \omega\rangle=-\nu_{0} \omega_{0}+\sum_{i=1}^{n+1} \nu_{i} \omega_{i}
$$

for all $\nu, \omega \in \mathbb{R}^{n+2}$. The $(n+1)$-dimensional de Sitter space $S_{1}^{n+1}$ is given by

$$
S_{1}^{n+1}=\left\{p \in \mathbb{R}_{1}^{n+2}:\langle p, p\rangle=1\right\} .
$$

Then it is easy to show that the metric induced from $\langle$,$\rangle turns S_{1}^{n+1}$ into a Lorentz manifold with constant sectional curvature one. We choose $\nu$ as an element of a canonical basis $a_{0}, \ldots, a_{n+1}$ of $\mathbb{R}_{1}^{n+2}$ and let $f_{A}$ and $g_{A}$ be the above functions for $\nu=a_{A}, A=0,1, \ldots, n+1$. Set

$$
f_{A}=\left\langle N, a_{A}\right\rangle, g_{A}=\left\langle x, a_{A}\right\rangle \text {. }
$$


Now observe that,

$$
\sum_{A=1}^{n+1} f_{A}^{2}=1+f_{0}^{2}, \sum_{A=1}^{n+1} g_{A}^{2}=1+g_{0}^{2}, \sum_{A=1}^{n+1} f_{A} g_{A}=f_{0} g_{0} .
$$

We define $\bar{x}=\int_{M} x d M$. Since $\langle x, x\rangle=1$, then it is elementary to conclude that $\langle\bar{x}, \bar{x}\rangle>0$. We choose $a_{0}=\frac{\bar{x}}{|\bar{x}|}$, then

$$
1=\left\langle a_{0}, a_{0}\right\rangle=-\left\langle a_{0}, x\right\rangle^{2}+\sum_{A=1}^{n}\left\langle a_{0}, e_{i}\right\rangle^{2}-\left\langle a_{0}, N\right\rangle^{2} \geq-g_{0}^{2}-f_{0}^{2} .
$$

Since $x$ is stable. Then, for each $A, J^{\prime \prime}(0)\left(g_{A}\right) \leq 0$. On the other hand,

$$
\begin{aligned}
& J^{\prime \prime}(0)\left(g_{A}\right) \\
& =\sum_{A=0}^{n+1} \int_{M}\left\{(r+1) L_{r}-a \Delta\right) g_{A}+(r+1)\left[(-1)^{r}(n-r) S_{r}\right. \\
& \left.\left.+(-1)^{r+1}\left(S_{1} S_{r+1}-(r+2) S_{r+2}\right)\right] g_{A}+a\left(n-S_{1}^{2}+2 S_{2}\right) g_{A}\right\} g_{A} d M \\
& =\int_{M}\left\{\left[(-1)^{r}(r+1) S_{r+1} f_{0} g_{0}-(-1)^{r}(n-r) S_{r} g_{0}^{2}\right]-a S_{1} f_{0} g_{0}+a n g_{0}^{2}\right. \\
& +(r+1)\left[(-1)^{r}(n-r) S_{r}+(-1)^{r+1}\left(S_{1} S_{r+1}-(r+2) S_{r+2}\right)\right] g_{0}^{2} \\
& +a\left(n-S_{1}^{2}+2 S_{2}\right) g_{0}^{2}-(-1)^{r}(n-r) S_{r}+a n+(r+1)\left[(-1)^{r}(n-r) S_{r}\right. \\
& \left.\left.+(-1)^{r+1}\left(S_{1} S_{r+1}-(r+2) S_{r+2}\right)\right]+a\left(n-S_{1}^{2}+2 S_{2}\right)\right\} d M \\
& \geq \int_{M}\left\{\left[(-1)^{r}(r+1) S_{r+1} f_{0} g_{0}-a S_{1} f_{0} g_{0}\right]-\left[(-1)^{r}(n-r) S_{r}-a n\right]\left(-1-f_{0}^{2}\right)\right. \\
& +(r+1)\left[(-1)^{r}(n-r) S_{r}+(-1)^{r+1}\left(S_{1} S_{r+1}-(r+2) S_{r+2}\right)\right]\left(-1-f_{0}^{2}\right) \\
& +a\left(n-S_{1}^{2}+2 S_{2}\right)\left(-1-f_{0}^{2}\right) \\
& -(-1)^{r}(n-r) S_{r}+a n+(r+1)\left[(-1)^{r}(n-r) S_{r}\right. \\
& \left.\left.+(-1)^{r+1}\left(S_{1} S_{r+1}-(r+2) S_{r+2}\right)\right]+a\left(n-S_{1}^{2}+2 S_{2}\right)\right\} d M \\
& =\int_{M}\left\{\left[(-1)^{r}(r+1) S_{r+1} f_{0} g_{0}-a S_{1} f_{0} g_{0}\right]-\left[(-1)^{r}(n-r) S_{r}-a n\right]\left(-f_{0}^{2}\right)\right. \\
& +(r+1)\left[(-1)^{r}(n-r) S_{r}+(-1)^{r+1}\left(S_{1} S_{r+1}-(r+2) S_{r+2}\right)\right]\left(-f_{0}^{2}\right) \\
& \left.+a\left(n-S_{1}^{2}+2 S_{2}\right)\left(-f_{0}^{2}\right)\right\} d M \\
& =-\int_{M} f_{0}\left((r+1) L_{r}-a \Delta\right) f_{0} d M
\end{aligned}
$$


$=\int_{M}\left(\left\langle P_{r} \nabla f_{0}, \nabla f_{0}\right\rangle-a\left\langle\nabla f_{0}, \nabla f_{0}\right\rangle\right) d M \geq 0$,

where we use Proposition 2.1, Lemma 4.1 and $a \leq 0$.

Therefore $J^{\prime \prime}(0)\left(g_{A}\right)$ must be zero. Then $\nabla f_{0}=0$ and $1+g_{0}^{2}=-f_{0}^{2}$. This implies that $g_{0}$ is constant and then $M$ is totally umbilical.

Acknowledgements. The author would like to thank the referees for giving some valuable suggestions which improved the paper. This project is supported by the National Natural Science Foundation of China (No. 61271002).

\section{References}

[1] H. Alencar, M. do Carmo, and A. G. Colares, Stable hypersurfaces with constant scalar curvature, Math. Z. 213 (1993), no. 1, 117-131.

[2] J. L. Barbosa and M. do Carmo, Stability of hypersurfaces with constant mean curvature, Math. Z. 185 (1984), no. 3, 339-353.

[3] J. L. Barbosa, M. do Carmo, and J. Eschenburg, Stability of hypersurfaces of constant mean curvature in Riemannian manifolds, Math. Z. 197 (1988), no. 1, 123-138.

[4] J. L. Barbosa and A. G. Colares, Stability of hypersurfaces with constant $r$-mean curvature, Ann. Global Anal. Geom. 15 (1997), no. 3, 277-297.

[5] J. L. M. Barbosa and V. Oliker, Stable spacelike hypersurfaces with constant mean curvature in Lorentz spaces, Geometry and global analysis (Sendai, 1993), 161-164, Tohoku Univ., Sendai, 1993.

[6] A. Brasil Jr. and A. G. Colares. Stability of spacelike hypersurfaces with constant r-mean curvature in de Sitter space, Proceedings of the XII Fall Workshop on Geometry and Physics, 139-145, Publ. R. Soc. Mat. Esp., 7, R. Soc. Mat. Esp., Madrid, 2004.

[7] F. Camargo, A. Caminha, M. da Silva, and H. de Lima, On the r-stability of spacelike hypersurfaces, J. Geom. Phys. 60 (2010), no. 10, 1402-1410.

[8] Q. M. Cheng, Complete space-like hypersurfaces of a de Sitter space with $r=a H$, Mem. Fac. Sci. Kyushu Univ. Ser. A 44 (1990), no. 2, 67-77.

[9] H. Cheng and X. Wang, Stability and eigenvalue estimates of linear weingarten hypersurfaces in a sphere, J. Math. Anal. Appl. 397 (2013), no. 2, 658-670.

[10] S. Y. Cheng and S. T. Yau, Hypersurfaces with constant scalar curvature, Math. Ann. 225 (1977), no. 3, 195-204.

[11] X. Liu and J. Deng, Stable space-like hypersurfaces in the de Sitter space, Arc. Math. 40 (2004), no. 2, 111-117.

[12] M. A. Velásquez, A. F. de Sousa, and H. F. de Lima, On the stability of hypersurfaces in space forms, J. Math. Anal. Appl. 406 (2013), no. 1, 134-146.

[13] H. Rosenberg, Hypersurfaces of constant curvature in space forms, Bull. Sci. Math. 117 (1993), no. 2, 211-239.

School of Mathematics and Statistics

JiAngSU Normal University

Xuzhou, Jiangsu 221116, P. R. China

E-mail address: zhangshicheng@jsnu.edu.cn 